\title{
Ultra-High Vacuum Aberration-Corrected STEM for in-situ studies
}

\author{
M.T. Hotz ${ }^{1}$, G.J. Corbin ${ }^{1}$, N. Dellby ${ }^{1}$, O.L. Krivanek ${ }^{1}$, C. Mangler ${ }^{2}$ and J.C. Meyer ${ }^{2}$ \\ ${ }^{1}$ Nion Co., 11511 NE $118^{\text {th }}$ St., Kirkland, WA 98034, USA \\ ${ }^{2}$ Dept. of Physics, University of Vienna, Boltzmanngasse 5, 1090 Vienna, Austria
}

Aberration-corrected scanning transmission electron microscopes (ACSTEMs) can resolve all the individual atoms, including impurities, in graphene and other 2D materials [1]. They can also record energy-dispersive X-ray spectra (EDXS) and fine-structure electron energy loss spectra (EELS) from individual atoms [2-4], and can operate at beam energies below the knock-on displacement threshold, thus minimizing or altogether avoiding radiation damage. These capabilities are allowing the structural, chemical, electronic and magnetic properties of matter to be examined in an unprecedented level of detail. They will become even more powerful if we can precisely control the sample environment and modify the sample structure in-situ.

Achieving these goals requires a flexible micro-laboratory inside the microscope, operating in ultrahigh vacuum (UHV). We have therefore developed a new sample chamber, shown in a side section in Fig. 1, for the Nion UltraSTEM [5]. The chamber is $120 \mathrm{~mm}$ high, a $60 \mathrm{~mm}$ increase relative to the regular sample chamber. The gap of its condenser-objective lens (OL) is $4 \mathrm{~mm}$, and the polepiece shape has been optimized for low chromatic aberration $\mathrm{C}_{\mathrm{c}}$ rather than low spherical aberration $\mathrm{C}_{\mathrm{s}}$ (which is corrected by the UltraSTEM's $\mathrm{C}_{3} / \mathrm{C}_{5}$ aberration corrector), with $\mathrm{C}_{\mathrm{c}}=1.0 \mathrm{~mm}$ and $\mathrm{C}_{\mathrm{s}}=1.1 \mathrm{~mm}$. The outer magnetic circuit was "bulked up" to allow for the extra magnetic flux due to longer polepiece pedestals, and the resultant configuration is capable of operating in a double crossover regime (with lowered OL aberrations coefficients) at $40 \mathrm{keV}$.

There are 3 new ports entering from the lower half of the chamber, at $25^{\circ}$ to the horizontal plane, with an unobstructed view of the entrance (lower) side of the sample. This means that they can be used for deposition or ion bombardment/etching of an untilted sample in the observation position at the center of the polepiece gap. The lower half of the chamber also has a custom-made Tisublimation pump of about 500 liters/s pumping capacity, and it provides space for a secondary electron detector (not shown). When the sample chamber has been baked at the maximum temperature of $140^{\circ} \mathrm{C}$, the Ti-sub pump should permit the vacuum at the sample to reach the $10^{-11}$ Torr range. A leak valve has also been provided, for immersing the sample in different gas atmospheres. The sample chamber is separated from the CFE gun of the microscope by two intermediate pumping volumes, which give about $10^{7}: 1$ differential pumping isolation. This arrangement readily allows operation with a sample chamber pressure of around $1 \times 10^{-5}$ torr. The upper half of the chamber mainly houses the microscope's 5-axis sample stage. It has 4 line-of-sight ports leading to the sample, which however cannot "see" an untilted sample. Six of the chamber's principal ports terminate in CF40 conflat flanges, which makes it possible to use conflat UHV windows and other standard vacuum attachments.

The sample stage can accept two very different types of sample holders (Fig. 2): the regular range of Nion side-insertion sample holders, which includes fixed tilt, double tilt and electrical/heating cartridges, or a $32 \times 15 \times 1 \mathrm{~mm}$ sample puck, which is inserted at $90^{\circ}$ to the regular sample port. A magnetically coupled UHV arm with a grabber allows the sample puck to be removed from the 
microscope and transferred to an auxiliary vacuum chamber. The auxiliary chamber, separate from the ACSTEM, provides facilities such as scanning tunneling microscopy (STM) [6], sample deposition, and other techniques of surface science.

The new system has recently been installed at the University of Vienna and is being tested. Results from the system will be reported at the meeting.

\section{References}

1. O.L. Krivanek et al., Nature 464 (2010) 571.

2. T.C. Lovejoy et al., Appl. Phys. Lett. 100 (2012) 154101.

3. W. Zhou et al. PRL 109 (2012) 206803; Q.M. Ramasse et al. Nano Lett. 13 (2013) 4989.

4. Y.-C. Lin et al., PRL 115 (2015) 206803.

5. O.L. Krivanek et al., Ultramicroscopy 108 (2008) 179.

6. http://www.dme-spm.com/special.html

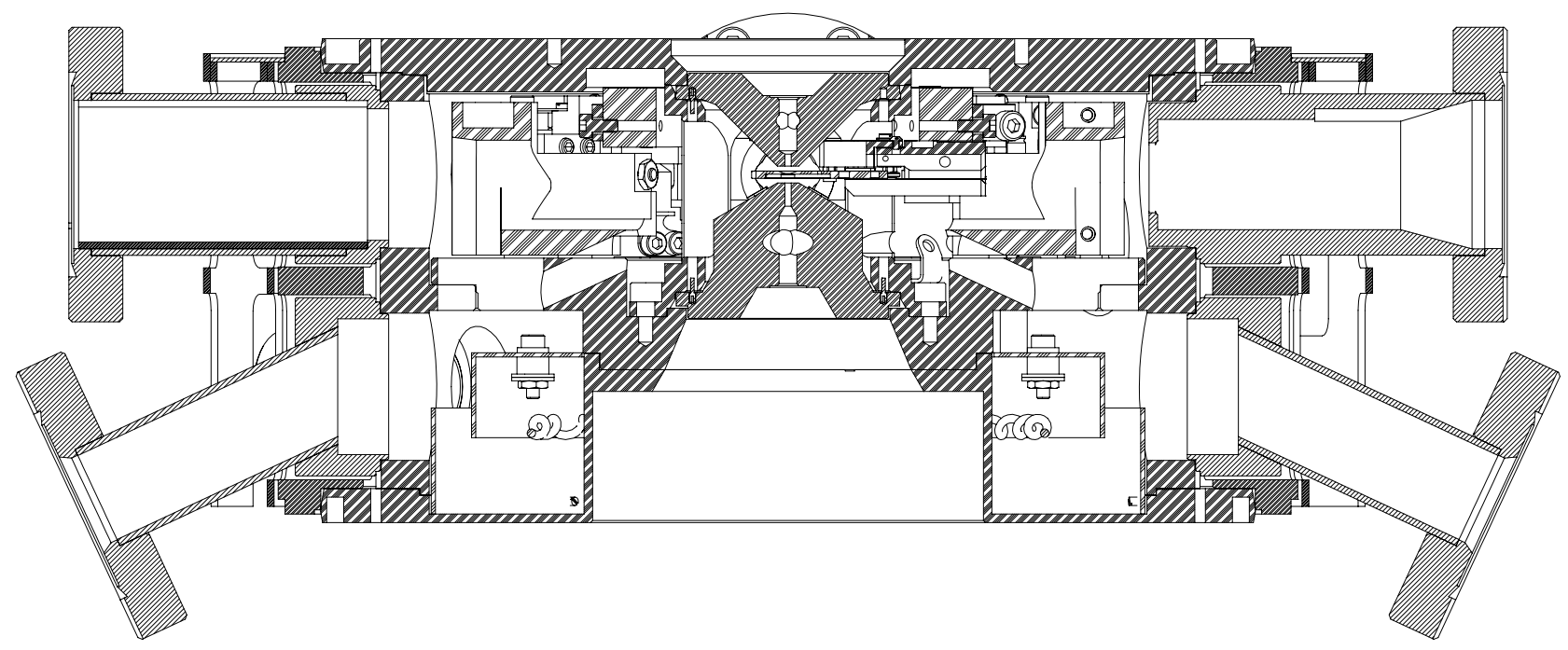

Figure 1. Cross-section of the UHV sample chamber, with an STM puck in the OL polepiece.
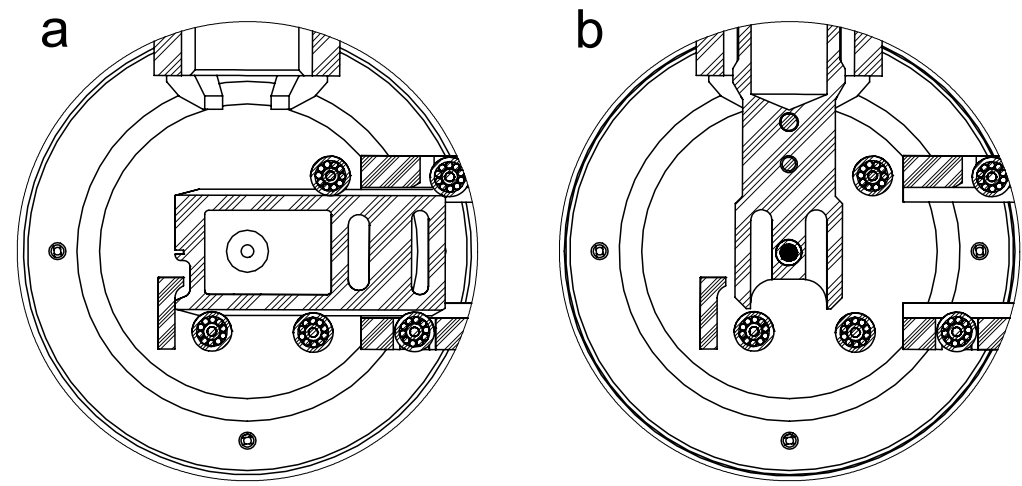

Figure 2. Horizontal cross-sections showing the OL region of the microscope operating with a) scanning tunneling microscope (STM) puck, b) regular Nion sample holder. 